\title{
Research on the Effect of Teaching Reform Based on Big Data of Achievement
}

\author{
Yang $\mathrm{Ni}^{1, \mathrm{a}}$ \\ ${ }^{I}$ School of Automation\&Electrical Engineering,Lanzhou Jiao Tong University,Lanzhou,China \\ a yangni@mail.lzjtu.cn
}

\begin{abstract}
Absrtact
Examination results are the most intuitive performance to test students' learning effect, and also an important feedback link of teaching effect. However, at present, many colleges and universities do not pay enough attention to the link of performance analysis, which leads to the disconnection of the feedback teaching mechanism of performance analysis. By using the big data of examination results, this paper analyzes the law of students' performance distribution, grasps students' learning effect, and deeply analyzes the correlation between teaching reform effect and performance analysis, so as to provide reference for the improvement of teaching mechanism and subsequent improvement in Colleges and universities.
\end{abstract}

Keywords: examination results, learning effect, teaching reform, big data

\section{基于成绩大数据的教学改革效果的分析研究}

\author{
杨妮 $1, \mathrm{a}$ \\ 1 兰州交通大学自动化与电气工程学院, 兰州甘肃 中国 \\ ayangni@mail.lzjtu.cn
}

\section{摘要}

考试成绩是检验学生学习效果的最直观表现, 也是教学效果的重要反馈环节。然而目前许多高校对成 绩分析环节不够重视, 导致成绩分析反馈教学机制脱节。利用考试成绩大数据, 分析学生成绩分布规 律, 掌握学生学习效果, 深入分析教学改革效果与成绩分析的相关性, 为高校教学机制完善及后续改 进提供参考。

关键词: 考试成绩, 学习效果, 教学改革, 大数据

\section{1. 前言}

考试作为检验学生学习效果的最直接方式, 也是教 学质量的最直观反馈。然而考试成绩分析却在实际教学 效果评价环节中未得到重视, 许多高效对学生成绩分析 只是蜻蜓点水, 粗略统计, 导致教学质量与教学效果的 验证徒有外表, 华而不实。考试成绩作为教学质量的重 要反馈环节, 挖掘大量学生成绩数据的规律及特点, 分 析其与教学质量两者之间的关系, 可以帮助教师改进教 学方法、提高教学效果, 了解学生的薄弱环节, 为后续 教学实施过程提供指导, 同时也可为学校教学管理者决 策提供借鉴。

\section{2. 数据来源}

\section{1 课程选取}

选取兰州交通大学轨道交通信号与控制专业《区 间信号自动控制》课程成绩数据进行分析。《区间信 号自动控制》课程是兰州交通大学轨道交通信号与控 制专业的核心专业课, 该课程实践性强, 内容知识点 众多, 旨在让学生系统地学习区间闭塞方法、掌握 Z PW-2000A 型移频自动闭塞原理及车站闭环电码化原 理。

\section{2 学生情况}

兰州交通大学轨道交通信号与控制专业 16 级共 5 个教学班, 分别为 1601 (茅以升班)、1602-1605 班, 其中 1601 班（茅以升班）是 16 级本专业高考学 习成绩前 30 名组成的班级, 在本级学习成绩相对较 好, 简称茅班, 其他班级均是成绩相当班级, 人数较 多, 简称普通班。选取 1605 班为教学改革班。各班 考场情况如表 1 所示。《区间信号自控控制》课程考 试总人数为 305 人, 取消考试资格 4 人, 最终实际考 试人数为 301 人。 
表 2 总评成绩情况统计表

兰州交通大学《区间信号自控控制》课程 2016 级 各教学班总评成绩评定办法为: 总评成绩=平时总成绩 (50\%) +期末考试成绩 $(50 \%)$ ，其中平时成绩=考勤成 绩（占总评成绩的 5\%) +作业成绩（占总评成绩的 1 $6 \%$ ）＋小测验成绩（占总评成绩的 $12 \%$ ）+实验成绩 (占总评成绩的 $12 \%$ ) +答 (质) 疑成绩 (占总评成绩 的 5\%)。本次 1605 班教学改革具体实施分为两部分 ${ }^{[1]}$ :

\section{表 1 考场情况统计表}

\begin{tabular}{|c|c|c|c|c|c|}
\hline 人数 & \multicolumn{5}{|c|}{ 班级 } \\
\hline 班级 & 1601 & 1602 & 1603 & 1604 & 1605 \\
\hline 考试人数 & 30 & 66 & 66 & 69 & 74 \\
\hline 缺考人数 & 0 & 0 & 0 & 0 & 0 \\
\hline 取消考试资格人数 & 0 & 0 & 4 & 0 & 0 \\
\hline 考场违纪人数 & 0 & 0 & 0 & 0 & 0 \\
\hline 考场情况 & 正常 & 正常 & 正常 & 正常 & 正常 \\
\hline
\end{tabular}

\section{1 教学过程}

首先强化实验预习思考环节, 加大故障案例, 深化 实验后总结分析能力, 提高学生的工程实践能力; 其次 延伸课堂学时, 引导学生积极利用课余时间, 充分利用 好现代化的信息工具和手段, 查找相关资料, 学习新知 识新技术理论, 补充课堂教学的局限性, 减少课堂教学 与铁路现场实际应用技术的脱节问题, 最后针对教学环 境的局限性, 提高教学课件的质量及简化学生的理解难 度, 丰富教学方法, 部分内容引入翻转课堂教学, 提高 学生学习的主观能动性。

\section{2 平时成绩考核}

针对课堂讲授过程, 一部分学生没有动手记笔记习 惯, 注意力不集中, 课后时间久课堂讲授的难点重点内 容遗忘情况, 鼓励学生动手做笔记, 记录每节课的难点 重点, 养成学习的好习惯。采取的措施是在原平时成绩 考核方式中减少考勤、答疑成绩分值, 加入课堂笔记成 绩项, 即: 平时成绩 $(50 \%)=$ 考勤成绩（占总评成绩的 $3 \%$ ）+作业成绩（占总评成绩的 16\%）+小测验成绩 （占总评成绩的 $12 \%$ ）+实验成绩（占总评成绩的 1 $2 \%$ ）+答（质）疑成绩（占总评成绩的 4\%) +课堂笔记 （占总评成绩的 $3 \%$ )。

\section{2016 级总评成绩分析}

《区间信号自控控制》 2016 级各教学班总评成绩情

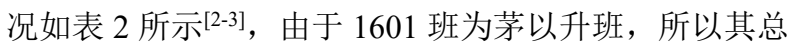
评成绩较其他普通版班最高, 普通班 1603 班总评成绩 平均值最低, 1602 班和 1605 班基本持平。

信号 16 级《区间信号自控控制》课程各教学班平

\begin{tabular}{|c|c|c|c|c|c|}
\hline \multirow{2}{*}{ 分数 } & \multicolumn{5}{|c|}{ 班级 } \\
\cline { 2 - 6 } & 1601 & 1602 & 1603 & 1604 & 1605 \\
\hline$\geq 90$ 分 & 0 & 2 & 0 & 2 & 1 \\
\hline $80-89$ 分 & 12 & 12 & 3 & 10 & 17 \\
\hline $70-79$ 分 & 13 & 17 & 15 & 14 & 25 \\
\hline $60-69$ 分 & 5 & 32 & 28 & 30 & 26 \\
\hline$<60$ 分 & 0 & 3 & 16 & 13 & 5 \\
\hline 平均分 & 76.1 & 71.4 & 63.17 & 65.81 & 71.5 \\
\hline
\end{tabular}

时成绩、试卷成绩、总评成绩平均值如表 3 所示。从 表 3 可以看到, 总评成绩平均值 1601、1602 和 1605 班较 1603、1604 班相对高, 均高于年级平均值 68 分。下面是针对《区间信号自控控制》课程各教学班 成绩总体细化分析。图 1 为《区间信号自控控制》 20 16 级各教学班平时成绩、考试成绩及总评成绩（60-1 00 分）人数的占各班总人数百分比情况, 从图 1 中 可以看到 1601 和 1602 班平时成绩均高于 60 分, 其 他班均有平时成绩不及格人数; 在考试成绩（60-100 分）的占比情况中，茅班 1601 接近 $83 \%$, 普通版 16 05 占比 $60 \%$ 左右, 其次是 1602 占比 $50 \%$ 左右, 1603 班相对占比较低。

\section{表 3 教学班教学情况统计表}

\begin{tabular}{|c|c|c|c|}
\hline \multirow{2}{*}{ 班级 } & \multicolumn{3}{|c|}{ 成绩 } \\
\cline { 2 - 4 } & 平时成绩 & 试卷成绩 & 总评成绩 \\
\hline $1601-1605$ (301 人) & 79.1 & 57.5 & 68 \\
\hline $1602-1605$ (271 人) & 79 & 55.9 & 67 \\
\hline
\end{tabular}

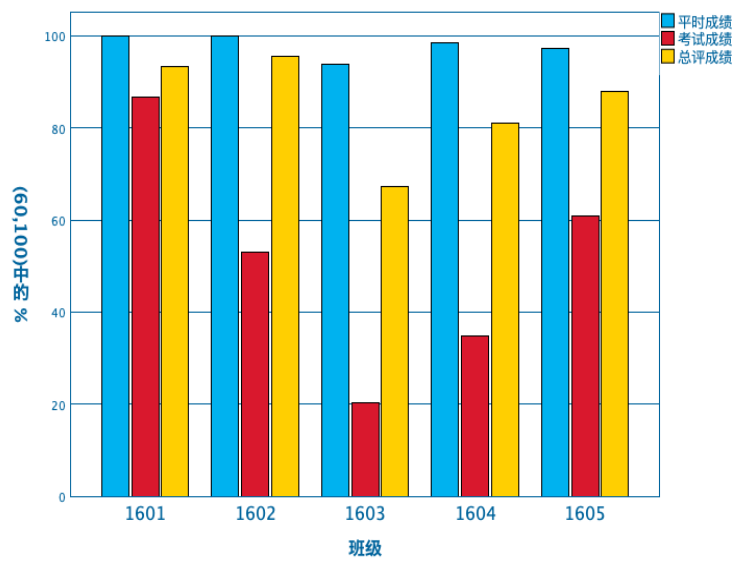

图 1 各教学班平时、考试及总评成绩(60-100 分) 人数百分比

图 2 为《区间信号自控控制》 2016 级各教学班 平时成绩、考试成绩及总评成绩各班的平均值情况。 可以看到茅班 1601 在平时成绩、考试成绩及总评成 绩三者之间相差较少; 普通版表现较好的是 1605 和 1602 班。 
表 4 各教学班试卷成绩统计表

三者之间的问题，平时成绩和考试成绩有明显的差异， 出现平时学习状态与考试成绩不一致。主要有以下几方 面:

（1）平时成绩的每项考核尺度存在差异。例如作 业、测试等环节存在作业题, 测试题不一致情况, 同时 每位任课教师的扣分尺度严格程度差异均可造成每班平 时成绩衡量水平差异。

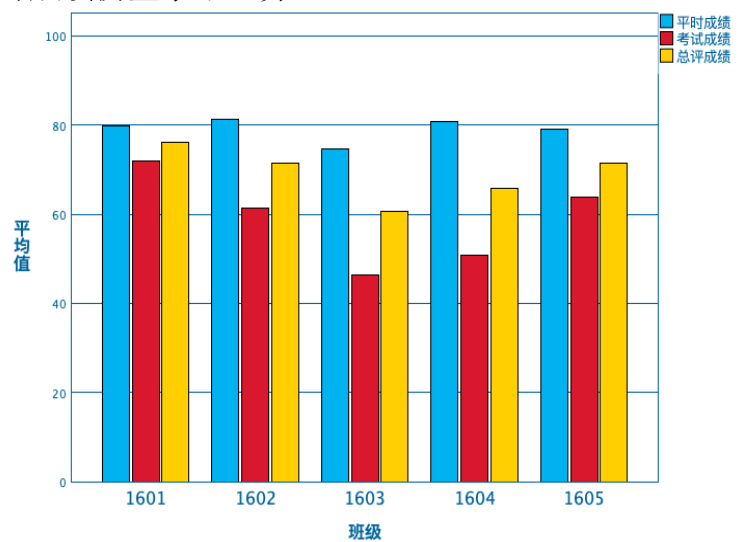

图 2 各教学班平时、考试及总评成绩平均值

（2）平时成绩存在虚假情况。由于作业、测试环 节等存在抄袭现象, 导致不能准确反映学生平时的学习 状态。

（3）教学效果不同。由于每班学生的整体学习风 气及每位学生的学习基础和态度直接影响教学效果的差 异, 例如茅班 1601 班总人数 30 人, 均是成绩较好学 生, 课堂教学效果整体表现较优。其他普通版人数差异 加之每班还有跟班重修的学生人数较多, 导致教学效果 班与班之间的差异。

针对以上情况, 应当加强平时成绩管理, 细化平时 成绩各项评分标准, 统一作业测试等考核内容, 尽量减 少虚假成绩（学生抄作业, 实验报告, 测验等) 的出 现, 杜绝不能体现学生真实学习状态行为的出现。同时 尽量小班教学, 提高教学效果, 减少平时成绩与考试成 绩之间的差异，做到公平公正。

\subsection{5 班《区间信号自控控制》课程改革效果 分析}

\section{1 试卷成绩分析}

《区间信号自控控制》课程 2016 级各教学班试卷 成绩统计如表 4 所示。可以看到, 高分少, 不及格率 高, 1605 班试卷成绩方面, 卷面成绩 90 分以上没有, 3 6.49\%学生成绩集中于 70-89.5 分, $24.32 \%$ 学生成绩集中 于 $60-69.5$ 分, $39.19 \%$ 学生成绩小于 60 分, 卷面不及格 率接近 $40 \%$, 在普通班里卷面成绩不及格率是最低的, 相对来说表现还是较好。

\begin{tabular}{|c|c|c|c|c|c|c|c|c|c|c|}
\hline \multirow{2}{*}{ 班级 } & \multicolumn{2}{|c|}{$\geq 90$} & \multicolumn{2}{|c|}{$80-89$} & \multicolumn{2}{|c|}{$70-79$} & \multicolumn{2}{|c|}{$60-69$} & \multicolumn{2}{|c|}{$<60$} \\
\cline { 2 - 11 } & $\begin{array}{c}\text { 人 } \\
\text { 数 }\end{array}$ & 百分比 & $\begin{array}{c}\text { 人 } \\
\text { 数 }\end{array}$ & 百分比 & $\begin{array}{c}\text { 人 } \\
\text { 数 }\end{array}$ & 百分比 & $\begin{array}{c}\text { 人 } \\
\text { 数 }\end{array}$ & 百分比 & 人数 & 百分比 \\
\hline 1601 & 1 & $3.33 \%$ & 8 & $26.67 \%$ & 10 & $33.33 \%$ & 6 & $20 \%$ & 4 & $13.33 \%$ \\
\hline 1602 & 1 & $1.52 \%$ & 6 & $9.09 \%$ & 13 & $19.70 \%$ & 15 & $22.73 \%$ & 31 & $46.97 \%$ \\
\hline 1603 & 0 & $0.00 \%$ & 1 & $1.64 \%$ & 5 & $8.20 \%$ & 7 & $11.48 \%$ & 49 & $79.03 \%$ \\
\hline 1604 & 0 & $0.00 \%$ & 2 & $2.90 \%$ & 12 & $17.40 \%$ & 10 & $14.50 \%$ & 45 & $65.20 \%$ \\
\hline 1605 & 0 & $0.00 \%$ & 13 & $17.57 \%$ & 14 & $18.92 \%$ & 18 & $24.32 \%$ & 29 & $39.19 \%$ \\
\hline
\end{tabular}

\section{2 改革效果分析}

本次考试涉及到与实践环节及翻转课堂讲授相关 的教学知识点在试卷中分别有体现, 现选取 1601

（茅班）和上面成绩分析普通版表现较好的 1602 班 与教改实施班 1605 班比较, 各知识点得分平均值如 表 5 及图 3 所示。

表 5 翻转课堂教学效果分析

\begin{tabular}{|c|c|c|c|c|c|}
\hline 知识点 & 试卷对应题目 & 分值 & 1601 班 & 1602 班 & 1605 班 \\
\hline $\begin{array}{c}\text { 发送器 } \\
\mathrm{N}+1 \text { 几余 }\end{array}$ & 一、5, 三、6 & 7 & 4.97 & 2.58 & 3.35 \\
\hline $\begin{array}{c}\text { 接收器双 } \\
\text { 机并联 }\end{array}$ & 五 & 6 & 5.07 & 4.95 & 5.27 \\
\hline $\begin{array}{c}\text { 闭环电码 } \\
\text { 化 }\end{array}$ & 一、6,8 & 6 & 4.73 & 2.91 & 4.03 \\
\hline
\end{tabular}

可以看到:

(1) ZPW-2000A 接收器双机并联知识点, 改革 班 1605 班平均得分 5.27 相比茅班 1601 班 5.07 分, 高出 $20 \%$, 相比普通版 1602 班高出 $32 \%$, 在三个班 里表现最好;

(2) 发送器 N+1 穴余知识点, 茅班 1601 班表 现较好平均分 4.97 分, 改革班 1605 班平均分 3.35 分 次之, 高出 1602 班 77\%;

(3) 闭环电码化知识点, 改革班 1605 班得分 4 . 03 分超出 1602 班 $112 \%$, 仅次于茅班 4.73 分。

ZPW-2000A 接收器双机并联、发送器 N+1 咒余 及闭环电码化均在翻转课堂和实验案例分析中有强 化, 通过以上分析可知, 本次教改效果明显, 学生分 数有很大提高。

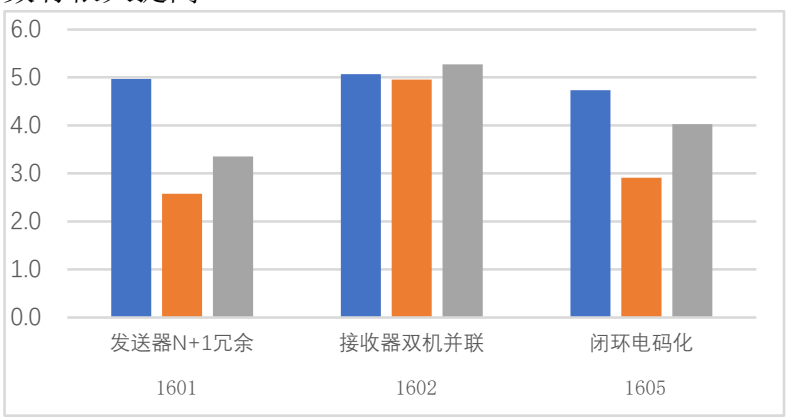

图 3 各知识点改革效果分析图 
在 16 级普通班里表现最优。

\section{1605 班《区间信号自控控制》课程试卷达成 情况分析}

《区间信号自控控制》课程教学大纲包含三个课程 目标, 本次试题共六道大题, 其题型有: 辨析题 (8个 小题共 24 分, 占 $24 \%$ )、电路分析题 (1 题共 14 分, 占 $14 \%$ )、设计及综合题（4题共 62 分，占 $62 \%$ )。1 1605 班 各课程目标达成情况如表 6 所示。

\section{表 61605 班试卷达成情况}

\begin{tabular}{|l|c|c|c|c|}
\hline 课程目标 & $\begin{array}{c}\text { 《区间信号自动控 } \\
\text { 制》试卷对应题号 }\end{array}$ & 应得分 & $\begin{array}{c}\text { 实际得分 } \\
\text { 平均值 }\end{array}$ & $\begin{array}{c}\text { 达成情 } \\
\text { 况值 }\end{array}$ \\
\hline 课程目标 1 & 一(1)、(2)、(3), 二 & 23 & 13.41 & 0.583 \\
\hline 课程目标 2 & $\begin{array}{c}\text { 一(4)、(5)、(6), 三 } \\
\text { (1)、(2)、(5)、 } \\
\text { (6)、(7), 四, 五 }\end{array}$ & 58 & 40.15 & 0.692 \\
\hline 课程目标 3 & 一(7)、(8), 三(3)、六 & 19 & 10.34 & 0.544 \\
\hline
\end{tabular}

注: 1. 实际得分平均值为该班对应各课程目标实际得分的班级 平均分值。2.达成情况值为实际得分平均值/应得分;

通过表 6 数据分析可知: 课程目标 1 最终评价结果 达到 58.3\%, 课程目标 2 最终评价结果达到 $69.2 \%$, 课 程目标 3 最终评价结果达到 $54.4 \%$, 课程目标 2 达到专 业毕业要求达成总体目标的要求, 课程目标 1 和课程目 标 3 略低于专业毕业要求达成总体目标的要求, 但综合 平时成绩后也能达到课程目标达成要求。1605 班各课程 目标达成情况雷达图如图 4 所示。可以看到课程目标 2 相对达成度较好。

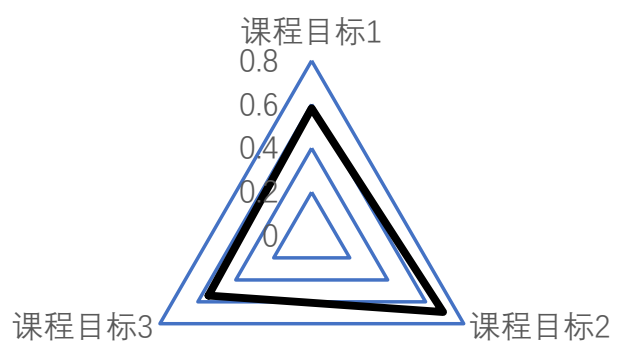

图 41605 班课程目标达成值雷达图

16 级各教学班三个课程目标达成平均值如图 6 所 示，可以看到课程目标 1 达成度较好的是茅班 1601 班, 其次是 1605 和 1604 班, 1602 班相对较低; 课程目 标 2 达成度较好的是茅班 1601 班，其次是 1605 和 1602 班, 1603 班相对较低; 课程目标 3 达成度较好的是茅班 1 601 班, 其次是 1605 班和 1602 班,1604 和 1605 班相对 较低。综合以上三个课程目标达成度可以看到 1605 班

\section{7. 总结}

通过对学生考试成绩数据的挖掘, 研究教学改革 效果与学生成绩之间的相关性, 得出:

(1)强化教学过程学生的主动性环节, 可以提高 学生对知识的掌握能力, 改变被动吸收为灵活运用, 提高教学质量。

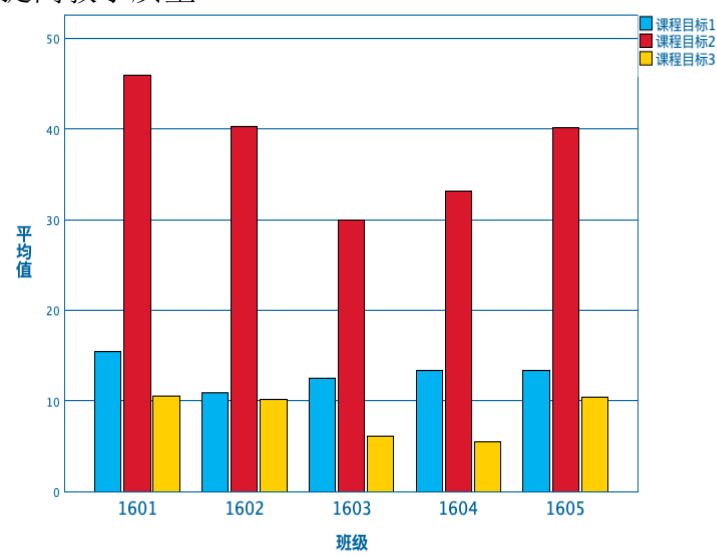

图 5 各教学班课程目标达成情况平均值

(2)不同班级的考试成绩具有不同的规律，这与 教学过程及学生的特点直接相关, 只有细化分析内部 及外部原因, 查找其中内在联系, 及时查漏补缺才能 达到较好的教学效果。

\section{项目基金}

本文为兰州交通大学教改研究课题《《区间信号 自动控制》课程教学模式的探索与实践》 (JGY20193 6）的阶段研究性成果之一。

\section{REFERENCES}

[1] Yang N. (2019) Teaching reform of "Automatic Interval Control of railway Signals" for railway specialty under the background of high speed railway development.Western China Quality Education, 24:182184.

[2] Xie H, Cai S. (2017) SPSS statistical analysis and data mining(Third Edition). Publishing House of Electronics Industry, Beijing.

[3] Wang Z. (2018) SPSS statistical analysis application case course. Peking University Press, Beijing. 